\section{CPS-052 FROM THE SURVEILLANCE OF ANTIBIOTICS PRESCRIPTIONS TO LIMITATION OF MULTIDRUG RESISTANT BACTERIA STRAINS: THE ROLE OF THE HOSPITAL PHARMACY IN DEFINING A CARBAPENEM-SPARING-STRATEGY}

L Gambitta*, E Galfrascoli, E Calzavara, V Curci, MLA Medaglia. ASST FatebenefratelliSacco- Fatebenefratelli and Ophthalmic Hospital, Hospital Pharmacy Operative Unit, Milan, Italy

\subsection{6/ejhpharm-2019-eahpconf.201}

Background The anti-microbial resistance (AMR) situation in Italy is worse than in many other EU Member States. The latest EARS-Net 2015 report confirms a high percentage of invasive bacterial isolates with disturbing AMR characteristics, significantly above the EU/EEA average. Klebsiella pneumoniae blood isolates resistant to carbapenems increased from $1.3 \%$ in 2006 to $33.5 \%$ in 2015 . For Escherichia coli, combined resistance increased from $0.8 \%$ in 2002 to $14.6 \%$ in 2015. Acinetobacter blood isolates resistant to carbapenems is very high: $83 \%$ (2012) and $78.3 \%$ (2015). The hospital pharmacy plays a major role in monitoring antibiotic prescriptions, in order to limit selection of the resistant bacteria strain.

Purpose Describe the pattern of antimicrobial prescribed with motivated request: focus on critical bacteria strains in order to define strategic intervention programmes.

Material and methods We collected data from the antibiotic prescriptions form June to September 2018. An Excel database was created. We focused on length of therapy, type of infection, antibiotic used, empiric versus target therapy and resistant bacteria strains.

Results We collected antibiotic prescriptions for 148 patients (55\% male, 45\% female) and 172 infections. Average age was 70.5 years' old. Average length of therapy was 10.5 days. Prevalent types of infection were: 23\% urinary tract infection (UTI); 22\% respiratory tract infections (RTI); 14\% sepsis; and $10 \%$ surgical site infections (SSI). Concerning critical bacteria strains (according to the WHO list): in 28\% (11/39) of UTI E. Coli ESBL+was isolated and treated with carbapenems; five Klebsiella Pneumoniae carbapenem-resistant were isolated (four from urinocolture treated with tygecicline, one from surgical site infections, which required treatment with ceftolozane/tazobactam $1.5 \mathrm{~g} \times 3$ for 2 weeks); one isolation of Acinetobacter baumanii MDR, carbapenem-resistant, responsible of complicated pneumonia, was treated with colistin; and three Pseudomonas Aeruginosa carbapenem-resistant, required treatment with ceftolozane/tazobactam and ceftazidime/avibactam, with clinical benefit. Of the total prescriptions 38\% were target therapy and 45\% empiric therapy: of 148 patients, 54\% were treated with carbapenems, $23 \%$ with quinolones and $15 \%$ with teicoplanin.

Conclusion Our data, although from a short period of time, confirms that carbapenems are the most prescribed antibiotics: their intensive use has contributed to bacteria resistant strains selections. Therefore, actual and future hospital priority is the improvement in a carbapenem-sparing-strategy, through post-prescription review and motivated request.

\section{REFERENCES AND/OR ACKNOWLEDGEMENTS}

ECDC country visit to Italy to discuss antimicrobial resistance issues.

No conflict of interest.

\section{CPS-053 STUDY ON DAPTOMYCIN USE AS A FIRST STEP TOWARDS AN ANTIMICROBIAL STEWARDSHIP PROGRAMME}

'S García Sánchez*, 'FJ García-Moreno, ${ }^{1} \mathrm{C}$ Rodríguez-González, ${ }^{1} E$ Chamorro-De Vega, ${ }^{1}$ E García-Martín, ${ }^{2} \mathrm{M}$ Valerio Minero, ${ }^{2} \mathrm{P}$ Muñoz-García De Paredes, ${ }^{3} \mathrm{E}$ Bouza-Santiago, ${ }^{1} A$ Herranz-Alonso, ${ }^{1} \mathrm{M}$ Sanjurjo-Sáez. ${ }^{1}$ Hospital General Universitario Gregorio Marañón, Pharmacy Department, Madrid, Spain; ${ }^{2}$ Hospital General Universitario Gregorio Marañón, Microbiology and Infectious Diseases, Madrid, Spain; ${ }^{3}$ Hospital General Universitario Gregorio Marañón, Instituto de Investigación Sanitaria Gregorio Marañón, Madrid, Spain

\subsection{6/ejhpharm-2019-eahpconf.202}

Background The use of daptomycin has increased in Spain since its approval and there is a great variability in the dosage and the criteria for its use in clinical practice.

Purpose This study was aimed at reporting the real use of daptomycin in clinical practice, including its efficacy and tolerability as a first measure for the establishment of improvement actions.

Material and methods Observational, retrospective study including all patients who started treatment with daptomycin during the period 1 January 2017 and 31 December 2017 in a Spanish tertiary hospital.

The following variables were collected: age, sex, comorbidities, type of infection, dosage, microbiological results, analytical parameters such as creatinine or creatine phosphokinase (CPK), adverse events and clinical outcome.

Results Overall, 176 patients (61.9\% men), median age of 70 years (IQR: 57-79), began treatment with daptomycin during 2017. The median of the Charlson comorbidity index was 3 (IQR: 2-6). Daptomycin was mainly used to treat skin and soft-tissue infections (37.5\%), fever without source (17.6\%), osteoarticular infection (12.5\%) and endocarditis (11.4\%). A total of 63 patients $(35.8 \%)$ presented a concomitant bacteremia.

Daptomycin was prescribed empirically in 58.0\% of patients. At the end of the follow-up, microbiological results were available in $89.2 \%$ of the total treated patients. Staphylococcus aureus was the most frequently isolated microorganism (58 microbiological isolates, only 10 resistant to methicillin). $43.8 \%$ of patients received doses $\leq 6 \mathrm{mg} / \mathrm{kg} /$ day, whereas $23.9 \%$ received doses $\geq 10 \mathrm{mg} / \mathrm{kg} /$ day. Infradosification was observed in at least 49 patients $(27.8 \%)$, who received doses $\leq 5 \mathrm{mg} / \mathrm{kg} /$ day. Daptomycin was administered for a median of 6 days (IQR: $3-13$ ). CPK was only monitored in $47.7 \%$ of patients treated with daptomycin for $\geq 7$ days.

Clinical evolution was satisfactory in $77.7 \%$ of patients. Total mortality was $17.6 \%$ and mortality related to the infection was $7.4 \%$. Five patients discontinued treatment due to adverse events (urticaria, cholestasis, increased CPK and rhabdomyolysis). No cases of resistance to the drug were reported.

Conclusion Daptomycin is a well-tolerated and effective drug but is often prescribed empirically or in infections not caused by $S$. aureus methicillin resistant. The follow-up of patients treated with daptomycin should be considered a priority intervention within the Antimicrobial Stewardship Programmes.

\section{REFERENCES AND/OR ACKNOWLEDGEMENTS}

PMID: 29261926.

No conflict of interest. 